A. K. Dupree and A. O. Benz, Eds.

\title{
The Sun as a Star: Comparing Alpha Cen A to UV Solar Spectra
}

\author{
Jeffrey L. Linsky \\ JILA/University of Colorado and NIST, Boulder, CO, USA
}

Isabella Pagano

Catania Astrophysical Observatory, Catania, Italy

Jeff A. Valenti

Space Telescope Science Institute, Baltimore, MD, USA

Doug Duncan

Dept. of Astrophysical and Planetary Sciences, University of Colorado, Boulder, CO, USA

\begin{abstract}
The Space Telescope Imaging Spectrograph (STIS) obtained high resolution echelle spectra of the nearby G2 V star $\alpha$ Centauri A covering the entire $1133-3150 \AA$ region with very high signal/noise. This data set provides what is probably the best approximation to the spectrum of the Sun viewed as a star, because it is a full disk spectrum with $2.6 \mathrm{~km} \mathrm{~s}^{-1}$ resolution, accurate absolute fluxes, full UV spectral coverage, high S/N, and low scattered light. In the 1140-1670 $\AA$ region we identify 671 emission lines from 37 different atoms and ions and the molecules $\mathrm{CO}$ and $\mathrm{H}_{2}$. We make a detailed comparison of the solar and $\alpha$ Cen A spectra in terms of line identification, line widths and Doppler shifts, emission measure distributions, and electron densities.
\end{abstract}

Surprising as it may appear, there is no high resolution UV spectral atlas of the Sun as a point source for direct comparison with stellar spectra obtained with STIS ${ }^{1}$. We need a solar spectral atlas with the following properties:

Full disk spectrum: It is extremely difficult to obtain a true full disk spectrum of the Sun. An accurate conversion from observed solar radiance (flux per steradian) to irradiance (point source flux) depends on properly accounting for center-to-limb properties, accurate spatial averaging, and intrinsic flux variability on solar cycle and short time scales.

Full UV spectral coverage: Most available solar spectra do not cover the full $1133-3150 \AA$ spectral range observable by STIS with very high S/N.

\footnotetext{
${ }^{1}$ Based on observations made with the NASA/ESA Hubble Space Telescope, obtained at the Space Telescope Science Institute, which is operated by AURA, under NASA contract NAS 5-26555. These observations are associated with proposal GO-07263.
} 
High spectral resolution: No available solar spectra approach the $2.6 \mathrm{~km} \mathrm{~s}^{-1}$ resolution of STIS.

Accurate absolute flux: The absolute flux scale of STIS is well calibrated ( $\pm 4 \%$ absolute) against well-studied white dwarf spectra.

Low scattered light: The STIS spectra are accurately corrected for instrumental scattered light using CALSTIS software.

We have therefore obtained a beautiful set of high resolution STIS spectra of the brightest star that is close in $T_{e f f}, \log g$, and metallicity to the Sun (see Table 1). Although the $m_{V}=5.5 \mathrm{mag}$ star $18 \mathrm{Sco}$ is thought to have nearly identical properties to the Sun, we have observed $\alpha$ Cen A because high resolution, high $\mathrm{S} / \mathrm{N}$ spectra are feasible in a modest $14 \mathrm{ks}$ of observing time.

Table 1. Stellar properties

\begin{tabular}{lcc}
\hline Parameter & Sun & $\alpha$ Cen A \\
\hline$T_{\text {eff }}$ & $5777 \mathrm{~K}$ & $5790 \pm 30 \mathrm{~K}$ \\
$g / g_{\odot}$ & 1.00 & 0.76 \\
$M / M_{\odot}$ & 1.00 & $1.16 \pm 0.03$ \\
$\log [M / H]$ & 0.00 & $\sim 0.20 \mathrm{dex}$ \\
$R / R_{\odot}$ & 1.00 & $\sim 1.2$ \\
$P_{\text {rot }}$ & 25.38 days & $\sim 22$ days \\
\hline \hline
\end{tabular}

We observed $\alpha$ Cen A on 1999 Feb 12 and report here on the E140H spectrum covering $1174-1687 \AA$ with $2.6 \mathrm{~km} \mathrm{~s}^{-1}$ resolution in TIME-TAG mode. The E230H spectrum will be discussed elsewhere. We reduced the data with CALSTIS Version 6.6 software, including the scattered light correction (IDL EHELLE_SCATT Routine). The nominal wavelength accuracy is $0.6-1.3 \mathrm{~km} \mathrm{~s}^{-1}$. For a full description of this program see Pagano et al. (2003).

We detected 671 emission lines in the $\alpha$ Cen A spectrum from 37 ions and the molecules $\mathrm{H}_{2}$ and $\mathrm{CO}$. A total of 172 of these lines are not seen in the existing SMM/UVSP (Shine \& Frank 2000; Woodgate et al. 1980) or SOHO/SUMER (Curdt et al. 2001) solar spectra at disk center, coronal hole, or a sunspot. The most highly ionized species detected are Ca VII $1261.07 \AA$, Si XII $1445.75 \AA$, and Fe XII $1242.00 \AA$. The He II $1640 \AA$ line is clearly weaker in $\alpha$ Cen A than the Sun, probably indicating that $\alpha$ Cen A has a cooler corona (see Fig. 1).

Broad wings are seen in the Si III $1206 \AA$, N V $1238 \AA$, Si IV 1393 and $1402 \AA, \mathrm{C}$ IV 1548 and $1550 \AA$ lines. Figure 2 shows two-Gaussian fits to these line profiles. Microflare heating (Wood et al. 1997) or "coronal funnels" (Peter 2001) may explain the broad components. Both the narrow and broad components are redshifted, although the narrow components are more redshifted as is observed for the Sun. The line centroids of $\alpha$ Cen A are slightly less redshifted than is found for the Sun (Fig. 3). However, the fraction of the flux in the broad components, $f_{B C} / f_{\text {tot } l}=0.46 \pm 0.05$, is similar to what is seen in active stars. The nonthermal velocities for these lines are similar to the Sun.

Analysis of the five O IV intersystem lines (1399-1407 $\AA$ ) yields $\log n_{e}=$ $9.8-10.2$, if $\log T_{e}=5.2$. The differential emission measure derived not using 


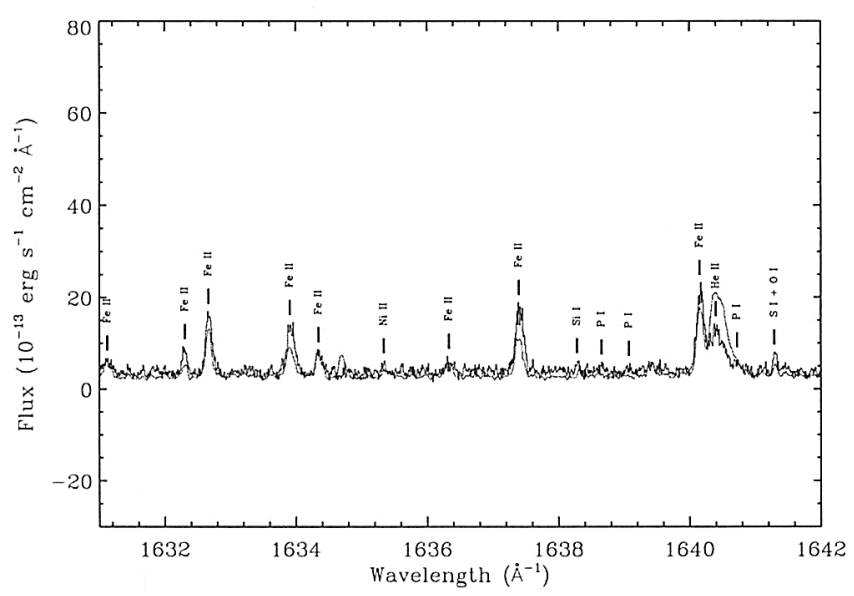

Figure 1. Comparison of a portion of the $\alpha$ Cen A STIS spectrum (thick line) containing the He II $1640 \AA$ line with the corresponding SMM/UVSP spectrum (thin line) observed with a $1 \times 180$ arcsec slit oriented N-S near solar disk center (Shine \& Frank 2000).

the $\mathrm{Li}$ and $\mathrm{Na}$ isoelectronic sequence ions is in close agreement with the quiet Sun (Landi \& Landini 1998), but is somewhat higher than for the quiet Sun at $30,000-100,000 \mathrm{~K}$ likely due to higher metal abundance of $\alpha$ Cen A.

Acknowledgement. This work is supported by NASA grant S-56500-D to NIST and the University of Colorado.

\section{References}

Curdt, W., Brekke, P., Feldman, U. et al. 2001, A\&A, 375, 591

Landi, E., \& Landini, M. 1998, A\&A, 340, 265

Pagano, I. et al. 2003, submitted to A\&A

Peter, H. 2001, A\&A, 374, 1108

Rassen, A.J.J. et al. 2003, A\&A, in press

Shine, R. \& Frank, Z. 2000, data retrivable from $\mathrm{ftp}: / /$ umbra.nascom.nasa.gov/pub/uv_atlases

Teriaca, L., Banerjee, D., \& Doyle, J.G. 1999, A\&A, 349, 636

Wood, B.E., Linsky, J.L., \& Ayres, T.R. 1997, ApJ, 478, 745

Woodgate, B.E. et al. 1980, PASP, 110, 1183 

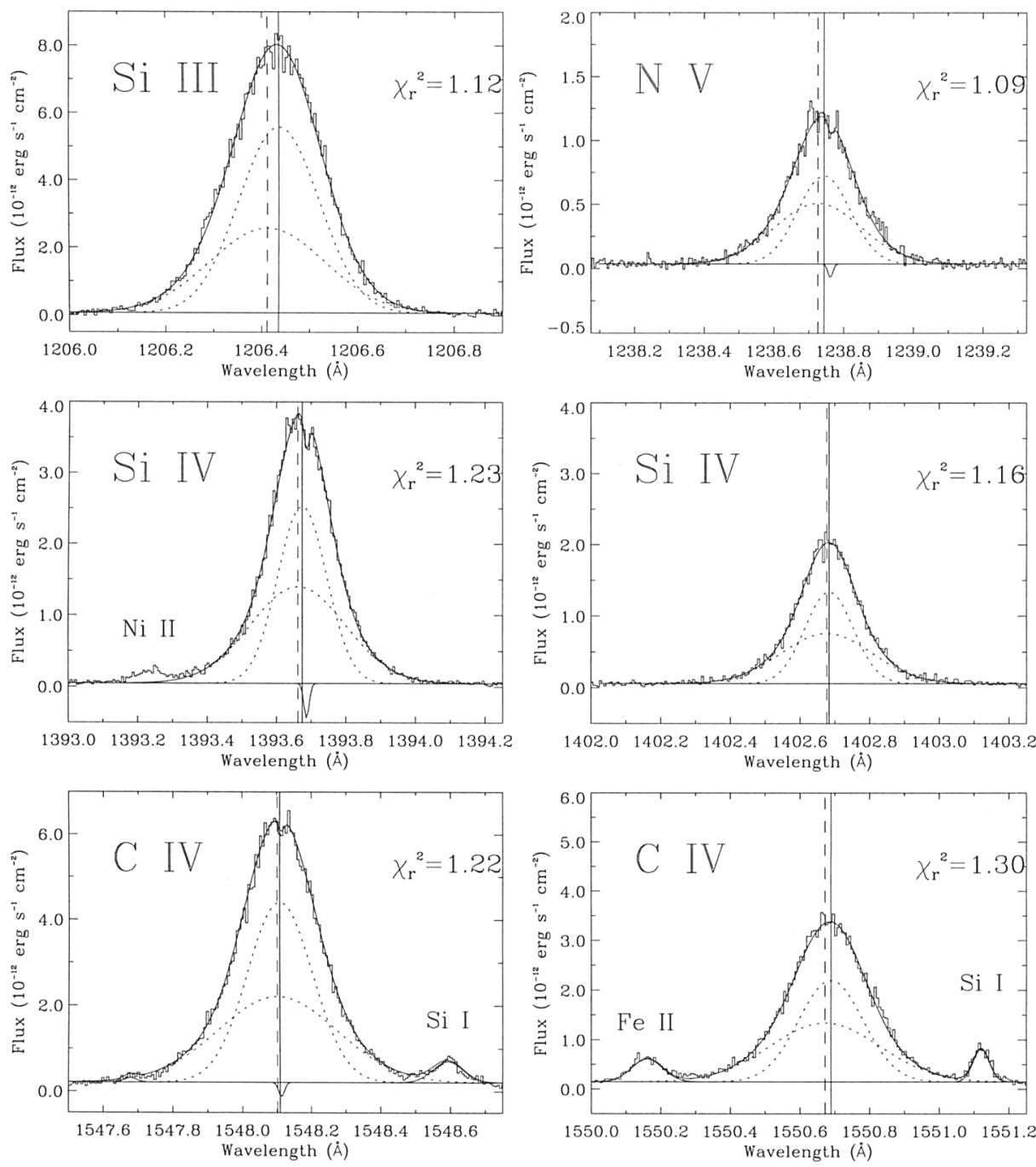

Figure 2. Comparison of observed and two-component model fits to important transition region lines of $\alpha$ Cen $\mathrm{A}$. The vertical solid and dashed lines indicate the centroids of the narrow and broad Gaussians, respectively, that are required to fit the observed line profiles. 


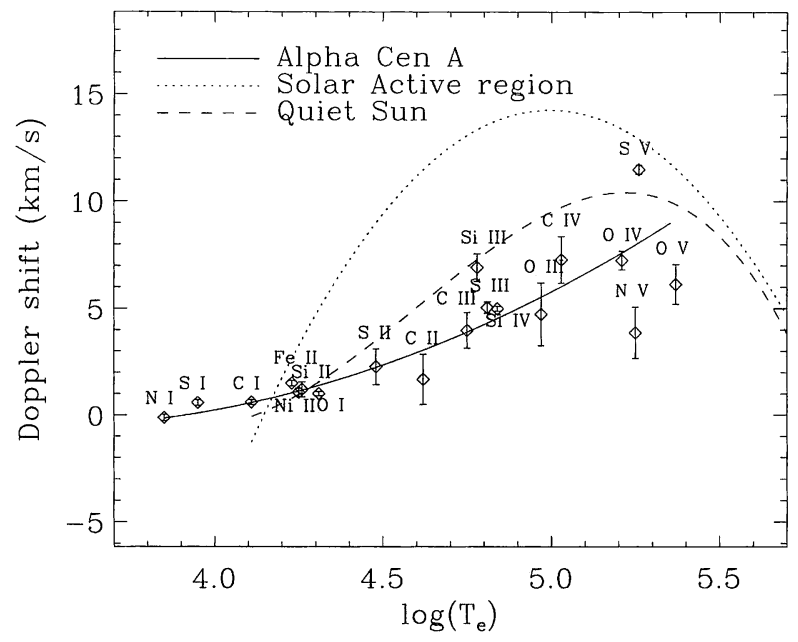

Figure 3. Doppler shifts of chromospheric and transition region lines of $\alpha$ Cen $\mathrm{A}$ relative to the photospheric radial velocity as a function of line formation temperature. The solid line is a fourth order polynomial fit to the data. The dotted and dashed lines are Doppler shifts for a solar active region and the quiet Sun, respectively (Teriaca et al. 1999).

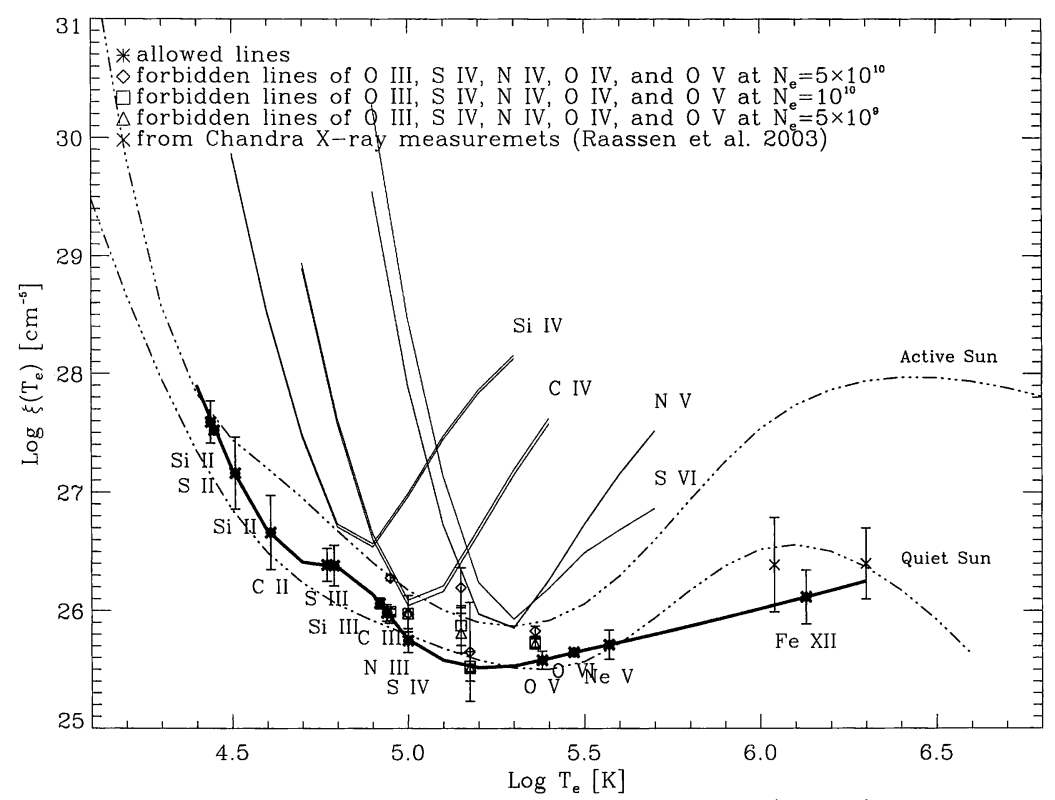

Figure 4. The differential emission measure (DEM) distribution of $\alpha$ Cen A (solid thick line) is compared with corresponding distributions of the quiet and active Sun. The intersystem lines of O III $1666 \AA$ and of the O IV multiplet near $1400 \AA$ match the DEM for $\log n_{e}=9.5-10$. The Chandra X-ray data are from Rassen et al. (2003). 


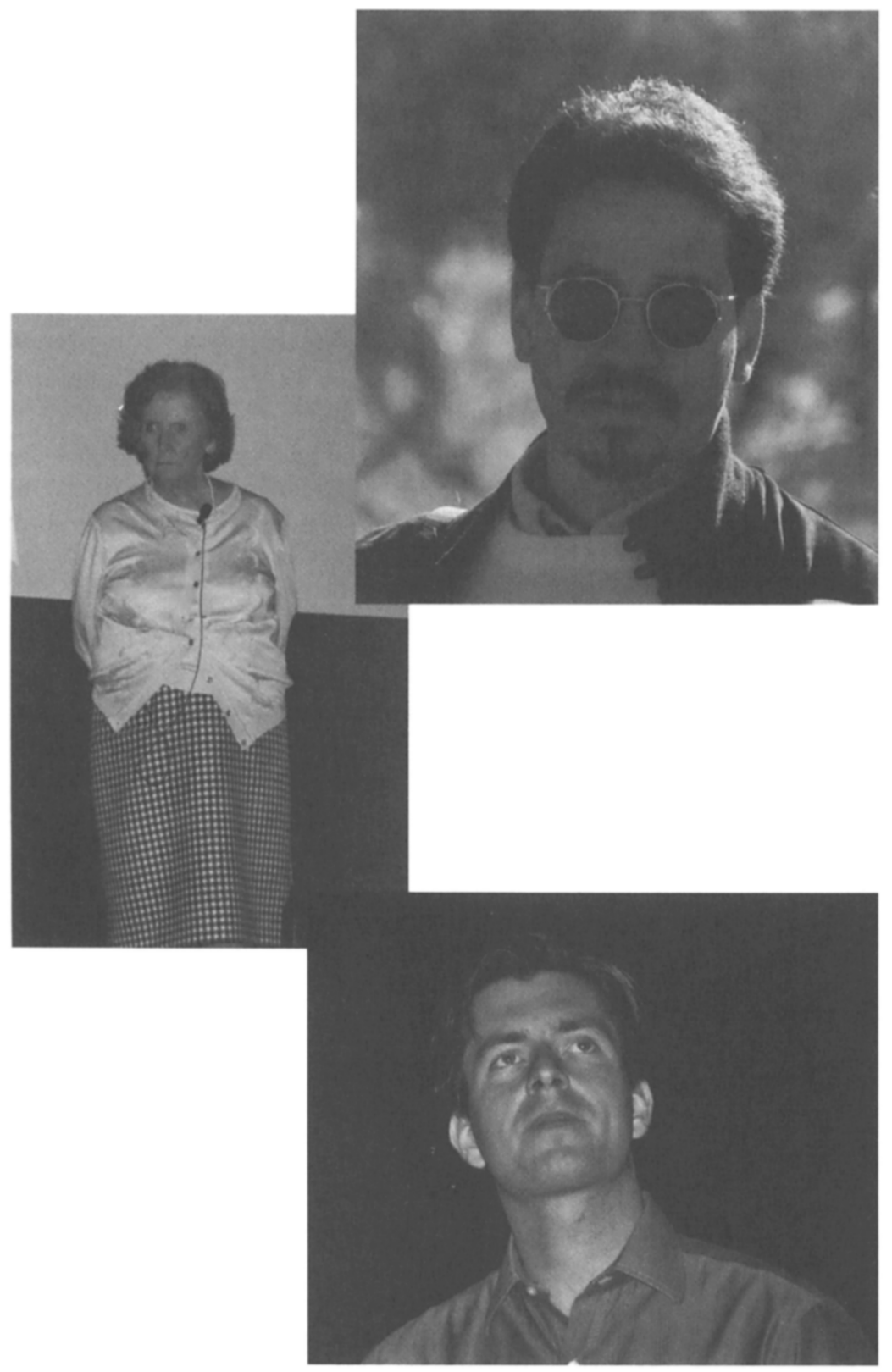

